http://dx.doi.org/10.32911/as.2018.v11.n2.587

Aporte Santiaguino 11(2), julio-diciembre 2018: 347-357

ISSN-L 2616-9541

\title{
Edificio multifamiliar antisísmico de 3 niveles de ferrocemento
}

\author{
Multifamily building with three levels of ferrocement \\ Ernesto Pérez Garezález ${ }^{1}$, Awaleh Kaireh Youssouf $^{1}$ y Xiomara Díaz Vega ${ }^{1}$
}

\section{RESUMEN}

El trabajo surge ante la necesidad de estudiar nuevos materiales que permitan construir viviendas resistentes de hasta tres niveles, dignas, seguras, económicas y sostenibles. Como es conocido el ferrocemento, mortero altamente reforzado con malla metálica ofrece amplias posibilidades en el cumplimiento de estas premisas.

Se parte de trabajos anteriores realizados en Cuba por los autores y se encamina el estudio, teniendo en cuenta la creciente demanda de viviendas de bajo costo y la escasez de ofertas en este sentido. Se realiza la modelación estructural teniendo en cuenta las características físico-mecánicas del ferrocemento y los demás materiales que intervienen en un edificio multifamiliar de viviendas de 3 plantas con paredes y piso de paneles nervados de ferrocemento de $3 \mathrm{~cm}$ de espesor así como la carga sísmica correspondiente a Cuba. La modelación incluye la discretización de la estructura en elementos finitos, lo que posibilita un estudio integral de los elementos de soportes y cierres, así como la evaluación de las conexiones entre ellos, después se realiza el diseño de los paneles de ferrocemento comprobándose ahorros sustanciales de materiales con relación a las viviendas tradicionales. Este constituye el punto de partida para el desarrollo de proyectos ejecutivos detallados y en él se estudian tipologías de viviendas que generarán el futuro desarrollo de sistemas constructivos.

Palabras clave: ferrocemento; estructura antisísmica; paneles nervados; modelación estructural; malla metálica.

\footnotetext{
1 Universidad de Camagüey. Camagüey, Cuba.

(C) Los autores. Este artículo es publicado por la Revista Aporte Santiaguino de la Universidad Nacional Santiago Antúnez de Mayolo. Este es un artículo de acceso abierto, distribuido bajo los términos de la Licencia Creative Commons Atribución-NoComercial-CompartirIgual 4.0 Internacional. (http://creativecommons.org/licenses/ by-nc-sa/4.0/), que permite el uso no comercial, distribución y reproducción en cualquier medio, siempre que la obra original sea debidamente citada.
} 


\begin{abstract}
The work is concibed for the necessity of studying new materials that allow to build resistant housings of up to three levels, worthy, sure, economic and sustainable. As the ferrocement, a mortar highly reinforced with metallic mesh, offers wide possibilities in the execution of these premises. It leaves of previous works carried out in Cuba by the authors and realize the study, keeping in mind the growing demand of housings of low cost and the shortage of offers in this sense. In this is carried out the structural modelation keeping in mind the physical-mechanical characteristics of the ferrocement and the other materials that intervene in a building of housings of 3 plants with walls and floor of nervouspanels of ferrocement of $3 \mathrm{~cm}$ of thickness as well as the seismic load corresponding to Cuba. The modelation includes the discretization of the structure in finite elements, what facilitates an integral study of the elements of supports and closings, as well as the evaluation of the connections among them, later is carried out the design of the ferrocement panels being proven substantial savings of materials with relationship to the traditional housings. This constitutes the starting point for the development of detailed executive projects and tipologys of housings is studied that will generate the future development of constructive systems.
\end{abstract}

Keywords: ferrocement; earthquake structural; nervous panels; structural modelation; metallic mesh.

\title{
INTRODUCCIÓN
}

Desde su surgimiento en el siglo XIX el Ferrocemento ha tenido múltiples usos tanto en barcos, muelles, patanas, depósitos entre otros.

La resistencia excepcional del ferrocemento se debe a que su armadura está compuesta por varias capas de mallas de acero de poco espesor superpuestas y ligeramente desplazadas entre sí, el hormigón soporta considerable deformación en la inmediata proximidad del refuerzo, condición que se aprovecha al máximo con la distribución de las armaduras descritas.

Su comportamiento mecánico, dependiente principalmente de la superficie específica de la armadura, es muy bueno. Presenta una buena resistencia a la tracción, que supera sensiblemente a la mostrada por el hormigón armado, y se mantiene en el rango elástico hasta su fisuración.

La presencia de las capas de mallas metálicas, no modifican la resistencia a la compresión, por lo que la misma específicamente queda definida por la resistencia a compresión del mortero que forma la matriz. 
Las viviendas de este material han demostrado que son una opción válida para enfrentar la crisis habitacional que hoy vive el mundo (Bedoya y Alvarez, 2009)

En Cuba desde la década de los 80 del pasado siglo se construyen viviendas de ferrocemento en varias provincias del país, en estas las paredes, los entrepisos y cubiertas han demostrado que soportan con eficiencia las cargas permanentes, uso y viento (Díaz y Pérez, 1988)

Sin embargo a pesar de varios intentos de especialistas cubanos de probar la carga sísmica en estructuras de ferrocemento no se ha desarrollado, en Cuba a nivel de diseño, proyectos de edificios de varias plantas de elementos portantes de ferrocemento.

La investigación tuvo como objetivo obtener distintos modelos estructurales aplicando técnicas digitales, para un edificio de 3 plantas multifamiliar sometido a diferentes sistemas de cargas incluyendo la carga sísmica.

Diseñar distintas variantes de elementos de soporte y cierre del edificio de ferrocemento con características sismorresistentes acorde a los resultados obtenidos en los modelos.

\section{MATERIALES Y MÉTODOS}

En la investigación que sustenta este trabajo se trabaja con ferrocemento como material estructural y por tanto se apela a las normativas vigentes en el país, la NC 2007. Código de buenas prácticas para el ferrocemento, además de las normas vigentes de la ACI (1988), como método de diseño para los paneles nervados.

En la investigación se utiliza la modelación estructural como método fundamental para simular digitalmente la estructura y las acciones que enfrenta. Se usa el Programa STAAD Pro. 2006, para modelar y cualificar el análisis de la carga sísmica, aplicando el Método de los elementos finitos en su ejecución.

\section{RESULTADOS Y DISCUSIÓN}

El proyecto consiste en un edificio de tres pisos. Se construirá con paneles nervados, las paredes portantes, la losa de entrepiso y la cubierta. Estas losetas prefabricadas de ferrocemento tienen $30 \mathrm{~mm}$ de espesor. Los cerramentos y la viga zapata son de hormigón armado, la altura de cada nivel sobre zapata es de 2,55 m. Este trabajo aborda el análisis de la edificación para los sistemas de cargas correspondientes y el diseño sólo de los elementos de pared. El análisis se hará para dos variantes una con conexión entre paneles y la otra variante sin conexión entre paneles. En la figura 1 se muestra la planta propuesta para este trabajo. 


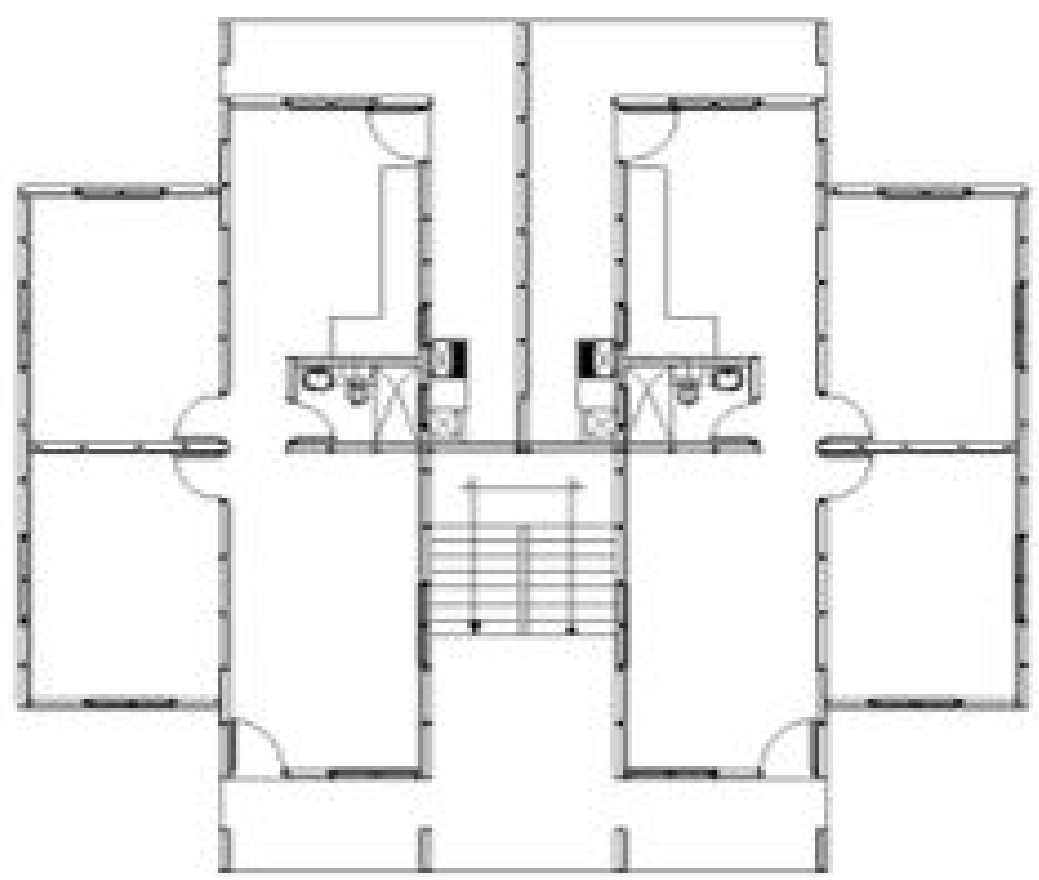

Figura 1. Planta del edificio de 3 niveles escogido como base de diseño (Díaz y Pérez, 2013)

Se trabajará con losas de pared con las dimensiones que aparecen en la tabla 1.

Tabla 1. Características y dimensiones de los elementos de pared escogidos

\begin{tabular}{|c|c|c|c|c|c|c|}
\hline \multirow[b]{2}{*}{$\begin{array}{c}\stackrel{0}{0} \\
\stackrel{0}{0} \\
\underset{0}{0} \\
\frac{0}{I I}\end{array}$} & \multicolumn{2}{|c|}{ Cantidad } & \multicolumn{2}{|c|}{ Dimensiones } & \multicolumn{2}{|c|}{ Nervio (mm) } \\
\hline & $\begin{array}{l}0 \\
0 \\
2 \\
0 \\
0 \\
0\end{array}$ & 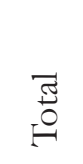 & Ancho & Largo & $\begin{array}{l}\frac{8}{\breve{U}} \\
\text { E्己 }\end{array}$ & $\begin{array}{c}\check{0} \\
\infty \\
0 \\
0 \\
0 \\
I\end{array}$ \\
\hline PF-1 & 41 & 123 & 700 & 2150 & 150 & 30 \\
\hline PF-2 & 16 & 48 & 1400 & 950 & 150 & 30 \\
\hline PF-3 & 4 & 12 & 700 & 1550 & 150 & 30 \\
\hline PF-4 & 49 & 147 & 900 & 2150 & 150 & 30 \\
\hline
\end{tabular}

En la figura 2 se muestra la sección de cada uno de los paneles que se usarán en el diseño. 

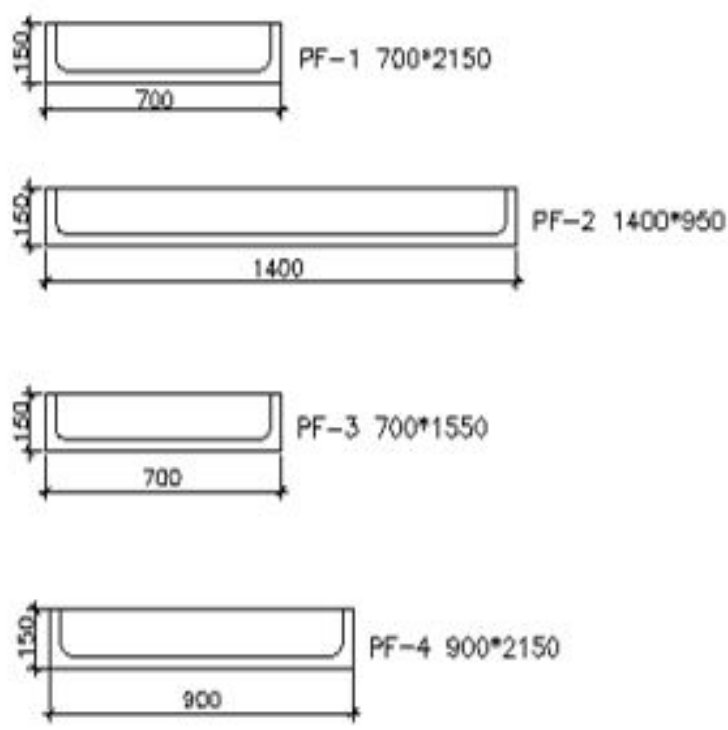

Figura 2. Sección de cada uno de los paneles

\section{Consideraciones para el diseño sísmico}

El edificio y cada una de sus partes deberá ser diseñado y construido para resistir las solicitaciones sísmicas de cálculo, de acuerdo con el riesgo sísmico de la zona, el tipo de perfil del suelo donde esté ubicado y con el tipo, uso e importancia de la obra.

En el método de análisis estático la acción sísmica se asimila a un sistema de fuerzas cuyos efectos sobre la estructura se calculan siguiendo los procedimientos de la estática (Llanes, 2005). Este sistema de fuerzas horizontales se aplica en el centro de masa de cada piso. El análisis separa el sismo en dos análisis independientes correspondientes a dos direcciones mutuamente perpendiculares.

El cortante basal de la estructura por piso aparece en la tabla 2, donde se considera el mismo para los dos ejes globales.

Tabla 2. Cortante basal por pisos en las dos direcciones

\begin{tabular}{ccccc}
\hline Pisos & Wi & Hi(m) & Wi*hi & F (kN) \\
\hline 3 & 837,67 & 7,65 & 6408,2 & 243,74 \\
2 & 1031,55 & 5,1 & 5260,9 & 200,10 \\
1 & 1031,55 & 2,55 & 2630,5 & 100,05 \\
\hline
\end{tabular}

El mismo se calculó con la fórmula (1):

$\mathrm{V}: \mathrm{AICW} / \mathrm{Rd}$

V: $0.3 \times 1 \times 2.5 \times 2900.80 / 4: 593,90 \mathrm{kN}$ 


\section{Las combinaciones de cargas usadas en el Staad Pro son:}

1,2 CP + 1,6 CU (ONN, 2003)

$0,9 \mathrm{CP}+1,4 \mathrm{CV}$

1,2 CP + 1,4 CE + 0,5 CU (para el sentido de X)

$1,02 \mathrm{CP}+1,4 \mathrm{CE}+0,5 \mathrm{CU}$ (para el sentido de Z)

\section{Modelación del edificio}

La estructura fue modelada en el programa STAAD. Pro 2006. Se trabaja en 2 modelos de análisis por el Método de los elementos finitos que son los siguientes:

-Entrepiso flexible con conectores entre paneles

-Entrepiso flexible sin conectores entre paneles

En cada uno de estos modelos se pedirá el informe al programa de los desplazamientos por pisos y por variantes y las tensiones más comprimidas y más traccionadas en cada eje global. En la figura 3 aparece la modelación geométrica del edificio

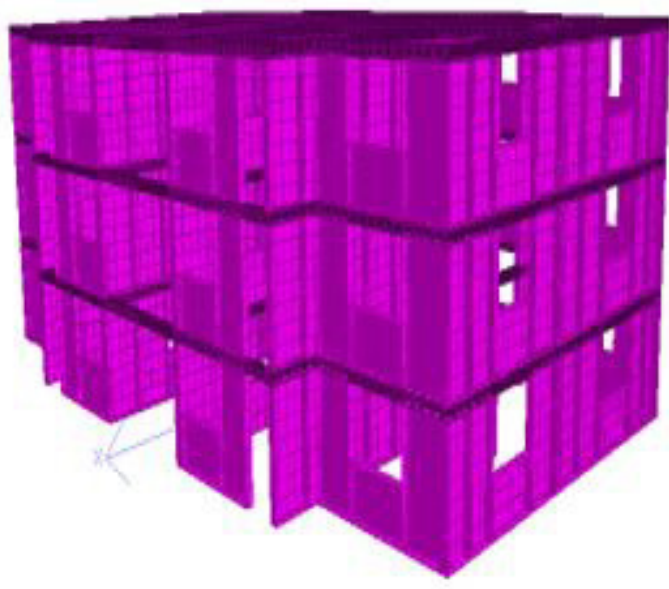

Figura 3. Modelo geométrico del edificio en STAAD Pro (Awaleh y Pérez, 2012)

Luego de modelada la estructura (figura 4) se escogen los valores máximos de desplazamiento por piso, en dirección de X y Z. En la tabla 3 se muestran los resultados en $\mathrm{X}$ y se compara este valor con el desplazamiento relativo permisible. De manera similar se hizo con Z, además se muestran las fórmulas comparativas de desplazamientos $\Delta=0,015 \mathrm{~h} / 4(2)$ y $\delta=\mathrm{H} / 600$ (3) donde h es la altura de cada piso y $\mathrm{H}$ es la altura total del edificio. 
Lo mismo se hizo para la variante de entrepiso flexible sin conexión entre paneles. En las dos variantes el desplazamiento es menor que el relativo permisible que es de 9,56mm y que el desplazamiento límite.

\section{Tensiones máximas en eje global $\mathrm{X}$ y $\mathrm{Z}$ en variante con conectores entre pane-} les.

Las tensiones máximas obtenidas a tracción en el eje $\mathrm{X}$ son de 2,53 MPa y a compresión 6,08 $\mathrm{MPa}$ ambas menores que $5 \mathrm{MPa}$ y $7 \mathrm{MPa}$, valores de tensiones que resisten los paneles de ferrocemento.

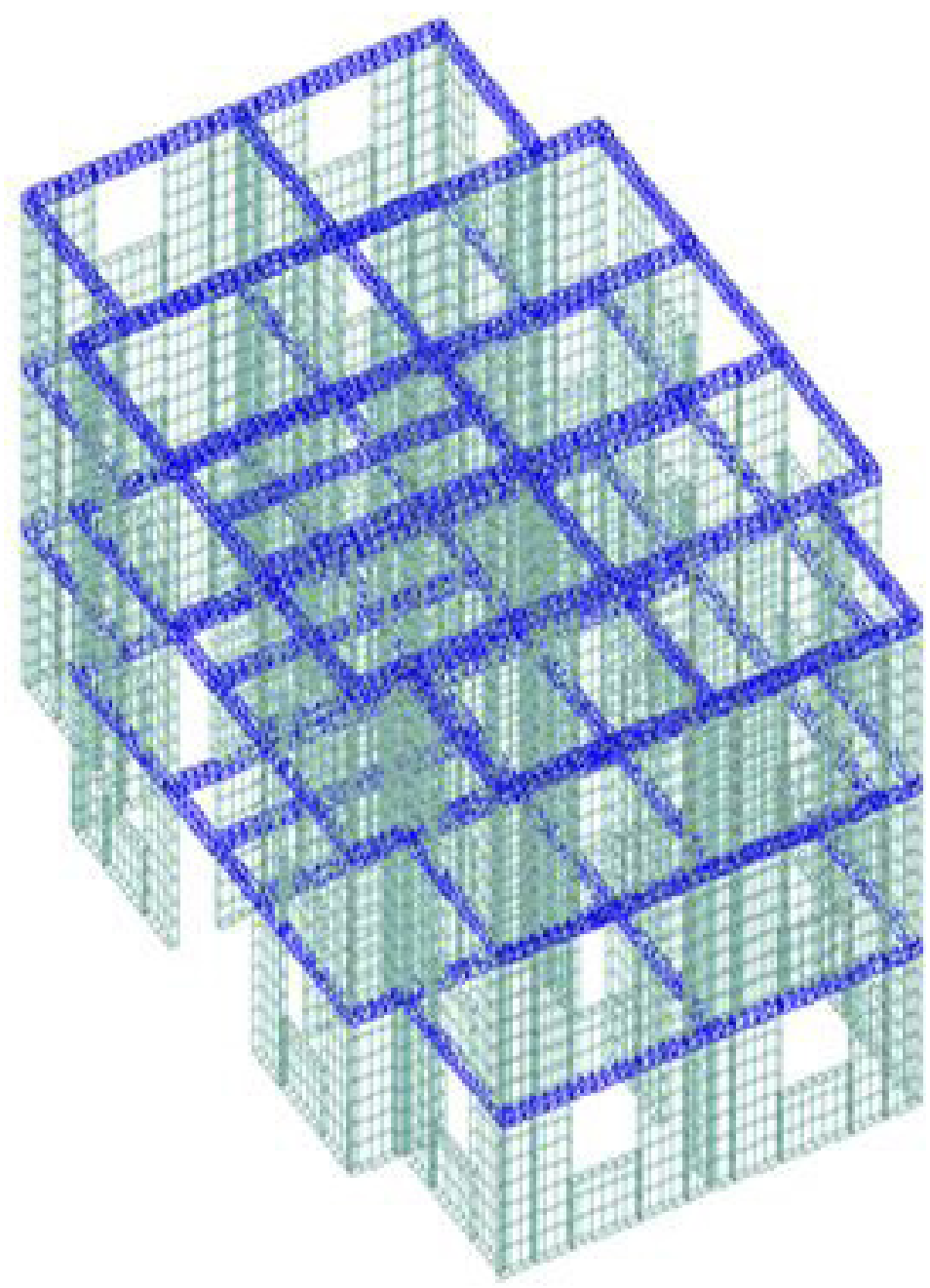

Figura 3. Paneles de ferrocemento en edificio modelado en el STAAD Pro 
Tabla 3. Desplazamiento máximo en dirección de X para modelo de entrepiso flexible con conectores entre paneles

\begin{tabular}{ccccc}
\hline Nivel & $\begin{array}{c}\text { Máximo } \\
\text { Desplazamiento } \\
(\mathrm{mm})\end{array}$ & $\begin{array}{c}\text { Desp. Max } \\
\text { en la est. } \\
(\text { con conector) }\end{array}$ & $\begin{array}{c}\text { Desp. relativo } \\
\text { Permisible }\end{array}$ & $\begin{array}{c}\text { Desp. } \\
\text { límite } \\
\delta=0,015 \mathrm{~h} / 4\end{array}$ \\
& & & & \\
& 3,578 & 0,001668 & & \\
\hline 3 & 1,910 & 0,000208 & 0,0095625 & 0,0125833 \\
2 & 1,702 & & & \\
\hline
\end{tabular}

De igual manera en el eje $Z$ se obtienen valores máximos a tracción de 4,43 MPa y de 6,18 $\mathrm{MPa}$ a compresión (Se obtuvo un valor aislado de 7,12 $\mathrm{MPa}$ que se considera muy cercano al permisible de $7 \mathrm{MPa}$ y se decide no tener en cuenta) (Llanes, 2005).

\section{Tensiones máximas en eje global $\mathrm{X}$ y $\mathrm{Z}$ en variante sin conectores entre paneles.}

Cumple en el eje X con valores de 2,69 MPa a tracción y 6,89 a compresión pero no cumple en el eje $Z$ ni en tracción con 7,86 MPa ni en compresión con 10,89 como se muestra en la figura 5 por lo que es necesario usar conectores para asimilar la tensión producida por la carga sísmica obtenida en el modelo estructural en las zonas con escotaduras (Llanes, 1995)

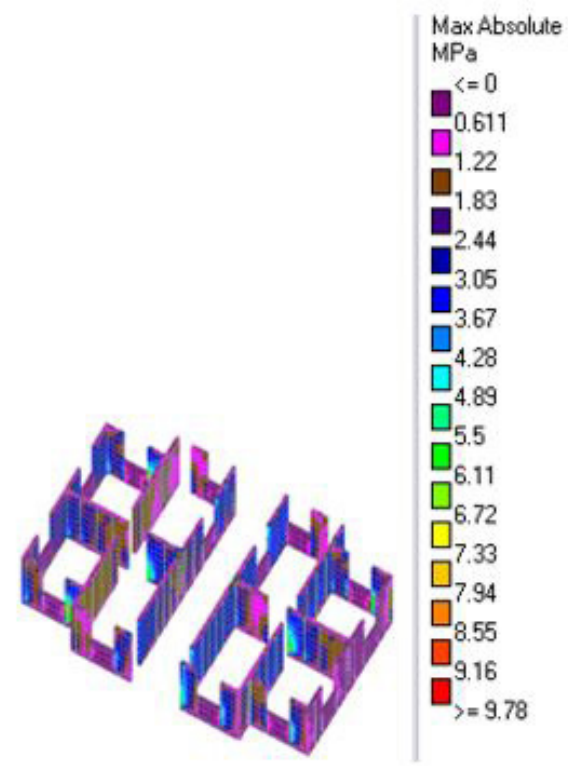

Figura 5. Tensiones en el eje Z (Díaz y Pérez, 2013) 


\section{Diseño de los elementos}

Se diseñan los cuatro elementos propuestos para tres tipos de malla, chequeando superficie específica $(\mathrm{S})$, fracción de volumen $\left(\mathrm{V}_{\mathrm{f}}\right.$ ) y fisuración $(\mathrm{W})$. El valor mínimo obtenido de $\mathrm{S}$ es de $0,66>0,5$. El valor mínimo de $\mathrm{V}_{\mathrm{f}}$ es de 4,21 \% > 1,8 \% y el valor máximo de fisuración obtenido fue de 0,05 para la malla tejida igual a la permisible por lo que se propone valorar casuísticamente su uso, la malla soldada y la hexagonal cumplen ampliamente pues el valor máximo es de 0,42

$\mathrm{S}=$ Area malla $/$ Vol acero malla $\left(\mathrm{cm} 2 / \mathrm{cm}^{3}\right)>0,5 \mathrm{~cm}_{(4)}^{-1}$

$\mathrm{V}_{\mathrm{f}}=($ Vrefuerzo / Vcompuesto $) \times 100>1,8 \%$ (5)

Wmáx $=(20 / \mathrm{Ea}) *(175+3,69(\delta$ at -345 S $)<0,05$ mm $(\mathrm{ONN}, 2007)$

En la tabla 4 aparecen las longitudes de los aceros utilizados como esqueleto en la conformación de los paneles.

Tabla 4. Longitudes de aceros por cada tipo de panel

\begin{tabular}{|c|c|c|c|c|c|c|}
\hline \multirow{2}{*}{ Elementos } & \multirow{2}{*}{ Tipo } & \multirow{2}{*}{$\varnothing(\mathrm{mm})$} & \multicolumn{2}{|c|}{ Dimensiones } & \multirow{2}{*}{$\begin{array}{l}\text { Long.(m) } \\
\text { unit }\end{array}$} & \multirow{2}{*}{ Cant. } \\
\hline & & & A & $\mathrm{B}$ & & \\
\hline \multirow{3}{*}{ PF-1 } & $\mathrm{BD}$ & 12 & 2,125 & 0,125 & 2,375 & 2 \\
\hline & BR & 12 & 2,125 & & 2,125 & 2 \\
\hline & $\mathrm{BR}$ & 6 & 0,675 & & 0,675 & 8 \\
\hline \multirow{3}{*}{ PF-2 } & $\mathrm{BD}$ & 12 & 0,925 & 0,125 & 1,175 & 4 \\
\hline & $\mathrm{BR}$ & 12 & 0,925 & & 0,925 & 2 \\
\hline & BR & 6 & 1,375 & & 1,375 & 5 \\
\hline \multirow{3}{*}{ PF-3 } & $\mathrm{BD}$ & 12 & 1,525 & 0,125 & 1,775 & 2 \\
\hline & $\mathrm{BR}$ & 12 & 1,525 & & 1,525 & 2 \\
\hline & $\mathrm{BR}$ & 6 & 0,675 & & 0,675 & 6 \\
\hline \multirow{3}{*}{ PF-4 } & $\mathrm{BD}$ & 12 & 2,125 & 0,125 & 2,375 & 3 \\
\hline & $\mathrm{BR}$ & 12 & 2,125 & & 2,125 & 2 \\
\hline & $\mathrm{BR}$ & 6 & 0,875 & 0,125 & 0,875 & 8 \\
\hline
\end{tabular}

En la tabla 5 se dan las cantidades de $\mathrm{m} 2$ de mallas necesaria por cada tipo de panel, utilizando dos capas. 
Tabla 5. Metros cuadrados de malla por panel

\begin{tabular}{cccccc}
\hline & \multicolumn{2}{c}{ Longitud Mallas $(\mathrm{m})$} & \multicolumn{2}{c}{ 2 capas de mallas $\left(\mathrm{m}^{2}\right)$} \\
\cline { 2 - 6 } & ancho & largo & exagonal & tejida & soldada \\
PF-1 & 0,925 & 2,375 & 4,39 & 4,39 & 4,39 \\
PF-2 & 1,625 & 1,175 & 3,82 & 3,82 & 3,82 \\
PF-3 & 0,925 & 1,775 & 3,28 & 3,28 & 3,28 \\
PF-4 & 1,125 & 2,375 & 5,34 & 5,34 & 5,34 \\
\hline
\end{tabular}

\section{CONCLUSIONES}

Se pre diseñaron 4 elementos de soportes y cierres y se modelaron en un edificio de tres plantas en dos variantes de estructura, una con conexión y la otra sin conexión entre paneles.

Se chequearon los momentos actuantes a partir de la tensión máxima obtenida en los resultados de la modelación dando por debajo las tensiones calculadas para cada tipo de paneles y de acuerdo a las mallas utilizadas.

El trabajo indica el uso de conectores para 3 plantas sobre todo en zonas de ventanas para contrarrestar la carga sísmica, carga que provoca los mayores desplazamientos y tensiones.

\section{REFERENCIAS BIBLIOGRÁFICAS}

ACI. 1988. «Guía para el diseño, construcción y reparación de ferrocemento». Structural Journal, 28 (4). New York, EU.

Awaleh, Kaireh y Pérez, E. 2012. Análisis y diseño de elementos portantes de ferrocemento antisísmico para edificio de viviendas de 3 plantas. Trabajo de diploma. Universidad de Camagüey, Cuba

Bedoya, D. y Álvarez, D. 2009. «Comportamiento de viviendas de ferrocemento para cargas cíclicas». Revista Ingenierías de Medellín, 8(15). Medellín, Colombia. 
Díaz, X y Pérez, E. 1988. Diseño de una casa de ferrocemento. Trabajo de Diploma no publicado. Universidad de Camagüey, Camagüey, Cuba.

Díaz, X y Pérez, E. 2013. Análisis y diseño de elementos portantes de ferrocemento para edificios multifamiliares antisísmicos. Tesis de Maestría. Universidad de Camagüey, Cuba.

Llanes, C. 1995. Análisis de un edificio de ferrocemento considerando los criterios para minimizar los efectos de un terremoto. Monografía. Ciudad de La Habana, Cuba: CECAT, ISPJAE.

Llanes, C. 2005. El ferrocemento una opción frente a los desastres [versión electrónica]. En Ponencia presentada en la IV Conferencia latinoamericana de ferrocemento. Recuperado el 24 de marzo de 2012 en http//www.cecat.com/ferrocemento

ONN. 2003. Norma Cubana 284. Edificaciones. Carga de Uso. La Habana, Cuba.

ONN. 2007. Código de buenas prácticas para el ferrocemento. La Habana, Cuba.

Recepción: 17/07/2018

Aceptación: 20/11/2018

Correspondencia

Ernesto Pérez Cerezález

ernesto.cerezalez@reduc.edu.cu 